\title{
Amplitude and Phase Statistics for Bistatic Scattering from Rough Surfaces
}

\author{
Kung-Hau Ding and William G. Stevens \\ Sensors Directorate, Air Force Research Laboratory \\ Hanscom AFB, MA 01731-2909 \\ Email: Kung-Hau.Ding@hanscom.af.mil
}

\begin{abstract}
In this paper we study the statistical characteristics of scattering from rough surfaces. By assuming normaldistributed and correlated real and imaginary scattering components, we derive analytical probability density functions (pdfs) for the amplitude and phase. The amplitude pdf is in the form of an infinite sum of modified Bessel functions while the phase pdf is expressed in terms of the error function. The analytical amplitude and phase distributions are verified by comparing with both the empirical distributions of simulated data and experimental measurements. Simulated data sets are derived from Monte Carlo simulations of bistatic scattering from ocean-like rough surfaces. Experimental data were collected from monostatic measurements conducted at a wind-roughened reservoir with an $S$-band polarimetric radar. These simulated and experimental results show that the amplitude and phase pdfs vary with wind speed, observation angle, and polarization. The TM wave is observed to have a wider amplitude distribution than a TE wave. The phase of the scattered field is not uniformly distributed, especially under the low wind condition. The analytical amplitude and phase distributions are in good agreement with the empirical distributions of both simulated and experimental data sets.
\end{abstract}

\section{INTRODUCTION}

Scattering of waves by random rough surfaces is an active research subject with applications in many disciplines of science and engineering [1-9]. It is known that the statistics of rough surfaces affect the characteristics of wave propagation and scattering. For natural surfaces such as water waves, environmental conditions play a dominant role in the surface realization, thereby affecting the scattered signal characteristics. Wind speed, in particular, directly affects surface roughness of water waves and should be considered in the statistical models of scattered signal components. Thus, it would be useful to study the distributions of bistatic scattering signals from rough surfaces under different environmental conditions. In this paper we extend the analytical work of [2] to derive analytical probability density function (pdf) models for both the amplitude and phase of scattered signals from rough surfaces. We obtain the amplitude pdf as an infinite sum of modified Bessel functions and the phase pdf expressed in terms of the error function. The advantage of these improved pdf models is that they require only the first and second moments of the received signals, i.e., the mean, variance, and correlation coefficient of the real and imaginary parts of the scattered field, which can be easily evaluated from measured data sets.

The analytical amplitude and phase distributions are verified by comparing with both the empirical distributions of simulated data and experimental measurements. Simulated data sets were derived from Monte Carlo simulations of bistatic scattering from one-dimensional randomly rough surfaces. The use of a physical surface spectrum model allows us to relate the observed statistical properties to geometric, environmental, and system considerations pertaining to the scattering surface. Experimental data were collected from monostatic measurements conducted at a wind-roughened reservoir with an $S$ band $(2.7 \mathrm{GHz})$ polarimetric radar [7]. These simulated and experimental results show that the amplitude and phase pdfs vary with wind speed, observation angle, and polarization. The TM wave is observed to have a wider amplitude distribution than a TE wave. The phase of the scattered field is not uniformly distributed, especially under the low wind condition. The analytical amplitude and phase distributions are in good agreement with the empirical distributions of both simulated and experimental data sets.

\section{Statistical Model of Scattered Field}

The field scattered from a random rough surface is a random process. The total scattered field $\psi_{s}$ is a sum of $N$ elementary scattered waves, which may be expressed as [3]

$$
\psi_{s}=\sum_{n=1}^{N} A_{n} e^{i \phi_{n}}=u+i v=\rho e^{i \chi}
$$

where $A_{n}$ represents the magnitude of the elementary scattered wave and $\phi_{n}$ the phase. In (1), $u$ and $v$ are the real and imaginary parts, and $\rho$ and $\chi$ are the amplitude and phase of the scattered signal, respectively. For large $N$, from the central limit theorem, $u$ and $v$ will follow approximately Gaussian distributions $[9,10]$. The joint probability density function, $p_{U V}(u, v)$, of $u$ and $v$ is given by the jointly Gaussian statistics:

$$
p_{U V}(u, v)=\frac{e^{-\frac{1}{2\left(1-r^{2}\right)}\left[\frac{\left(u-u_{0}\right)^{2}}{\sigma_{u}^{2}}-2 r \frac{\left(u-u_{0}\right)\left(v-v_{0}\right)}{\sigma_{u} \sigma_{v}}+\frac{\left(v-v_{0}\right)^{2}}{\sigma_{v}^{2}}\right]}}{2 \pi \sigma_{u} \sigma_{v}\left(1-r^{2}\right)}
$$

where $u_{0}=\langle u\rangle, v_{0}=\langle v\rangle, \sigma_{u}^{2}=\left\langle\left(u-u_{0}\right)^{2}\right\rangle, \sigma_{v}^{2}=$ $\left\langle\left(v-v_{0}\right)^{2}\right\rangle$, and $r=\frac{\left\langle\left(u-u_{0}\right)\left(v-v_{0}\right)\right\rangle}{\sigma_{u} \sigma_{v}}$ is the correlation coefficient [10]. The angular brackets $\langle\cdot\rangle$ denote the ensemble 
average. Making the variable transformations leads to an expression for the joint pdf for the amplitude and phase,

$p_{R X}(\rho, \chi)=\frac{\rho e^{-a} e^{\rho(s \cos \chi+t \sin \chi)-\rho^{2}(b+c \cos 2 \chi+d \sin 2 \chi)}}{2 \pi \sigma_{u} \sigma_{v} \sqrt{1-r^{2}}}$,

where the parameters $a, b, c, d, s$, and $t$ are given as functions of $u_{0}, v_{0}, \sigma_{u}, \sigma_{v}$, and $r$, respectively.

$$
\begin{aligned}
a & =\frac{u_{0}^{2} \sigma_{v}^{2}+v_{0}^{2} \sigma_{u}^{2}-2 r u_{0} v_{0} \sigma_{u} \sigma_{v}}{2 \sigma_{u}^{2} \sigma_{v}^{2}\left(1-r^{2}\right)} \\
b & =\frac{\sigma_{u}^{2}+\sigma_{v}^{2}}{4 \sigma_{u}^{2} \sigma_{v}^{2}\left(1-r^{2}\right)} \\
c & =\frac{\sigma_{v}^{2}-\sigma_{u}^{2}}{4 \sigma_{u}^{2} \sigma_{v}^{2}\left(1-r^{2}\right)} \\
d & =\frac{-r}{2 \sigma_{u} \sigma_{v}\left(1-r^{2}\right)} \\
s & =\frac{u_{0} \sigma_{v}-r v_{0} \sigma_{u}}{\sigma_{u}^{2} \sigma_{v}\left(1-r^{2}\right)} \\
t & =\frac{v_{0} \sigma_{u}-r u_{0} \sigma_{v}}{\sigma_{u} \sigma_{v}^{2}\left(1-r^{2}\right)} .
\end{aligned}
$$

Integrating (3) with respect to $\chi$, the amplitude pdf is obtained as an infinite sum of modified Bessel functions

$$
\begin{aligned}
p_{R}(\rho)= & \frac{\rho e^{-a-b \rho^{2}}}{\sigma_{u} \sigma_{v} \sqrt{1-r^{2}}}\left[I_{0}\left(\sqrt{c^{2}+d^{2}} \rho^{2}\right)\right. \\
& I_{0}\left(\sqrt{s^{2}+t^{2}} \rho\right)+2 \sum_{m=1}^{\infty}(-1)^{m} \\
& I_{m}\left(\sqrt{c^{2}+d^{2}} \rho^{2}\right) I_{2 m}\left(\sqrt{s^{2}+t^{2}} \rho\right) \\
& \left.\cos \left(2 m\left(\tan ^{-1} \frac{d}{c}+\tan ^{-1} \frac{t}{s}\right)\right)\right]
\end{aligned}
$$

where $I_{m}(\cdot)$ is the $m^{\text {th }}$ order modified Bessel function of the first kind. Note that $p_{R}(\rho)$ is a function of the correlation coefficient $r$. Assuming $r=0$ and $\sigma_{u}=\sigma_{v}=\sigma_{0}$, the amplitude pdf reduces to the Rice distribution. If the scattered field is totally diffuse, such that $\left\langle\psi_{s}\right\rangle=0$, the amplitude distribution becomes Rayleigh.

Integrating (3) with respect to $\rho$, we obtain the phase distribution function

$$
p_{X}(\chi)=\frac{e^{-a}\left\{1+\sqrt{\pi} \xi e^{\xi^{2}}[1+\operatorname{erf}(\xi)]\right\}}{4 \pi \sigma_{u} \sigma_{v} \sqrt{1-r^{2}}(b+c \cos 2 \chi+d \sin 2 \chi)},
$$

where $\operatorname{erf}(\cdot)$ is the error function, and

$$
\xi=\frac{s \cos \chi+t \sin \chi}{2 \sqrt{b+c \cos 2 \chi+d \sin 2 \chi}} .
$$

Here, too, $p_{X}(\chi)$ is a function of the correlation coefficient $r$. In the case of totally diffuse scattering, the phases become uniformly distributed, $p_{X}(\chi)=\frac{1}{2 \pi}$, over the range 0 to $2 \pi$.

\section{Bistatic Scattering Statistics}

In this section, we compare the analytical amplitude and phase pdfs with both the empirical distributions of simulated data and experimental measurements. Simulated data sets are derived from Monte Carlo simulations of bistatic scattering from one-dimensional rough surfaces. Experimental data were collected from experiments conducted at a wind-roughened reservoir located in central Massachusetts with an $S$-band polarimetric radar [7].

\section{A. Simulated Data from Ocean-Like Surfaces}

The statistics of bistatic scattering from ocean-like surfaces under various wind conditions are presented here. The sea scattering simulations are performed by using the PiersonMoskowitz (P-M) spectrum. The one-dimensional form of the $\mathrm{P}-\mathrm{M}$ spectrum is given by [4]

$$
W(|k|)=\frac{a_{0}}{4|k|^{3}} \exp \left\{-\frac{\beta g^{2}}{|k|^{2} U^{4}}\right\},
$$

where $a_{0}=0.0081, \beta=0.74, g=9.81 \mathrm{~m} / \mathrm{s}^{2}$, and $U$ is the wind speed, in $\mathrm{m} / \mathrm{s}$, at the altitude of $19.5 \mathrm{~m}$ above the mean sea level. The P-M ocean-like surface has a multiscale roughness controlled by the upper and lower cutoff frequencies, $k_{U}$ and $k_{L}$, for the spectrum, which are given as $k_{L}=0.75338 \mathrm{rad} / \mathrm{m}$ and $k_{U}=418.879 \mathrm{rad} / \mathrm{m}$ in generating the P-M surfaces. For each wind speed, 2000 independent surface samples are created based on the P-M spectrum. The Banded Matrix Iterative Approach (BMIA) [4] technique is applied to numerically compute bistatic scatterings from rough surfaces. Each rough surface is of length $L=6.144 \mathrm{~m}$ and is divided into $N=2048$ intervals. The permittivity of sea water is equal to $59.08+i 34.87$. A tapered incident wave is used to minimize the truncation errors caused by surface boundaries and the tapering parameter is set to be $\frac{L}{4}$. The radar frequency and incidence angle are fixed at $10 \mathrm{GHz}$ and $\theta_{i}=30^{\circ}$. Both TE and TM modes are considered.

To check the amplitude and phase distributions, the histograms of simulated sea returns are compared with the theoretical models of (10) and (11), respectively. Figures 1 and 2 present the amplitude and phase distributions for backscattered signals $\left(\theta_{s}=-30^{\circ}\right)$ and $U=0.5$ and $5 \mathrm{~m} / \mathrm{s}$, respectively. The top row is for the TE case and the bottom row for the TM case. The empirical histogram responses are compared with the theoretical distributions. As can be seen from these figures, the matches are quite good. For the amplitude pdf, TM shows a wider distribution than TE. The phase appears to be non-uniformly distributed. For the low wind speed case of $U=0.5 \mathrm{~m} / \mathrm{s}$, the phase distribution shows two profound peaks. The peaks in the phase distribution diminish as wind speed increases, $U=5 \mathrm{~m} / \mathrm{s}$, such that the surface becomes rougher. Figure 3 shows the amplitude and phase distributions with regard to forward scattering $\left(\theta_{s}=30^{\circ}\right)$. It is interesting to note that, for the low wind speed case in Figure 3, the amplitude distribution shows a longer "left" tail instead of a "right" tail as in the other cases. In addition, the phase is distributed over a very narrow range due to the small roughness caused by low wind speed. In Table I, we summarize the mean, standard deviation, and mean-to-standard deviation ratio, $\frac{\langle\rho\rangle}{\sigma_{\rho}}$, for the 


\begin{tabular}{|c|c||c|c||c|c|}
\hline \multicolumn{2}{|c|}{ Polarization } & \multicolumn{2}{c|}{ TE } & \multicolumn{2}{c|}{ TM } \\
\hline$U$ & $\theta_{s}$ & $-30^{\circ}$ & $30^{\circ}$ & $-30^{\circ}$ & $30^{\circ}$ \\
\hline \hline & $\langle\rho\rangle$ & 3.744 & 598.0 & 8.461 & 753.6 \\
\hline 0.5 & $\sigma_{\rho}$ & 2,347 & 14.85 & 5.264 & 19.65 \\
\hline & $\frac{\langle\rho\rangle}{\sigma_{\rho}}$ & 1.608 & 40.26 & 1.607 & 38.35 \\
\hline \hline & $\langle\rho\rangle$ & 5.034 & 68.67 & 10.37 & 86.64 \\
\hline 5 & $\sigma_{\rho}$ & 3.080 & 36.15 & 5.973 & 45.53 \\
\hline & $\frac{\langle\rho\rangle}{\sigma_{\rho}}$ & 1.635 & 1.899 & 1.736 & 1.903 \\
\hline
\end{tabular}

TABLE I

MEAN, STANDARD DEVIATION, AND MEAN-TO-STANDARD DEVIATION RATIO FOR TE AND TM WAVES.
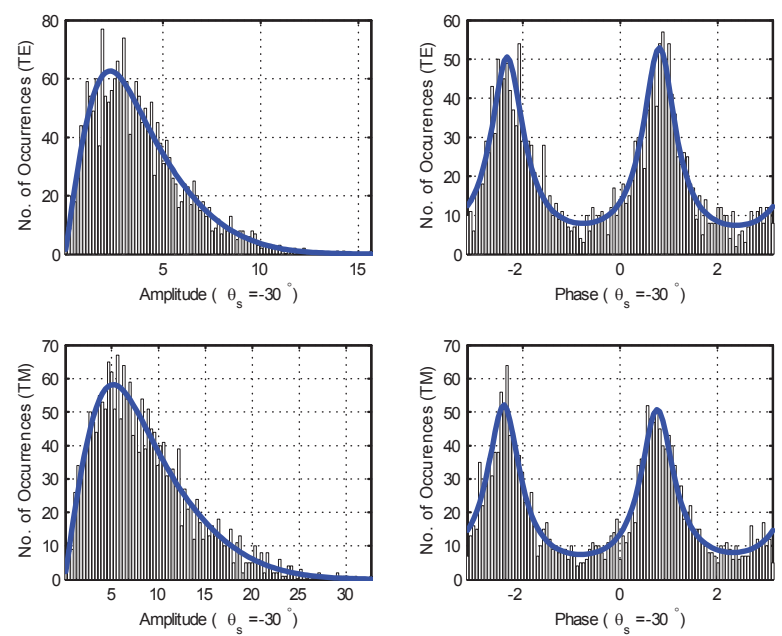

Fig. 1. Amplitude and phase distributions for $\mathrm{U}=0.5 \mathrm{~m} / \mathrm{s}$ and $\theta_{s}=-30^{\circ}$.

amplitude distribution evaluated from the simulated data for the cases considered in Figures 1-3. We can see that the mean of the amplitude for the TM mode is larger than that for TE, which implies that the scattering coefficient of TM is larger than TE. The standard deviation of the amplitude for the TE mode is smaller than that for TM, which indicates that TE has a more narrow amplitude distribution than TM. The ratios of $\frac{\langle\rho\rangle}{\sigma_{\rho}}$ are smaller than 1.913, a constant characterizing the Rayleigh pdf. This indicates that the data distribution has a tail longer than that of a Rayleigh distribution. For both TE and $\mathrm{TM}$ at $U=0.5 \mathrm{~m} / \mathrm{s}$ and $\theta_{s}=30^{\circ}$, the values of $\frac{\langle\rho\rangle}{\sigma_{\rho}}$ are quite large, 40.26 for TE and 38.35 for TM. From these results we can see that the simulated sea return data are characterized by a non-Rayleigh distribution and the statistical distributions of amplitude and phase vary with wind speed, observation angle, and polarization.

\section{B. Measurement Data from Reservoir}

In this section we compare the experimental monostatic data with the analytical pdf models. The experiments were conducted at a reservoir located in central Massachusetts. The $S$-band $(2.7 \mathrm{GHz})$ radar system used for this data set employed simple pulse transmission to achieve resolution in range, which was approximately $15 \mathrm{~m}$. A $1.2 \mathrm{~m}$ parabolic reflector antenna
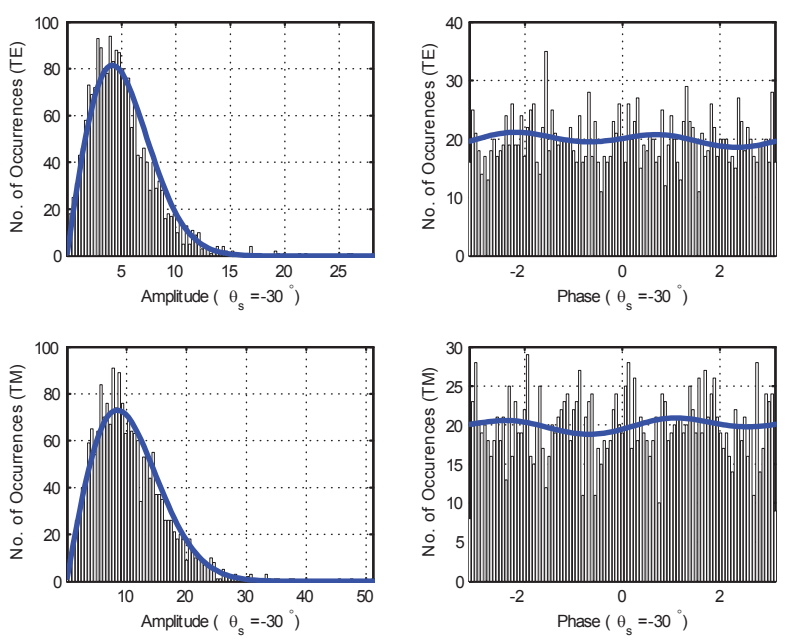

Fig. 2. Amplitude and phase distributions for $\mathrm{U}=5 \mathrm{~m} / \mathrm{s}$ and $\theta_{s}=-30^{\circ}$.
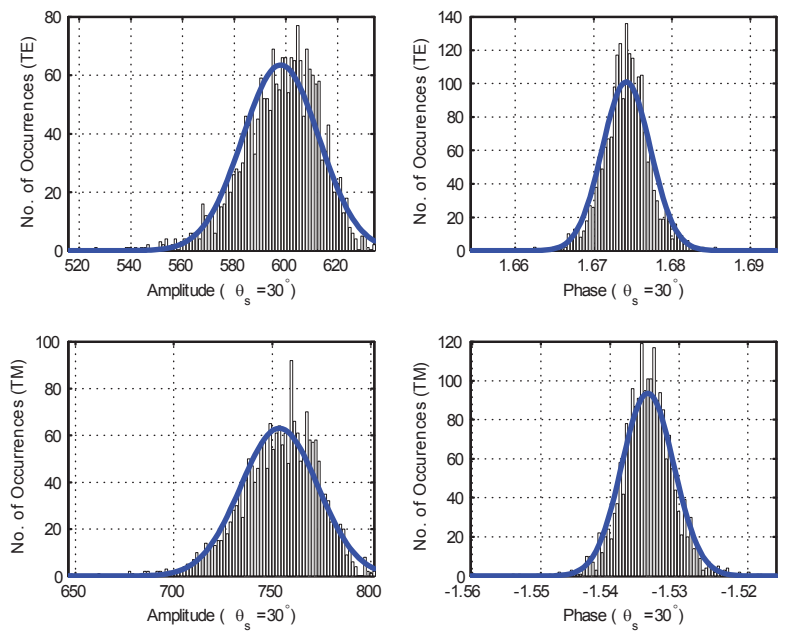

Fig. 3. Amplitude and phase distributions for $\mathrm{U}=0.5 \mathrm{~m} / \mathrm{s}$ and $\theta_{s}=30^{\circ}$.

with a dual-polarized feed was used both for transmitting and receiving the vertically and horizontally polarized signals. The radar system was installed in a stationary truck located near the edge of the reservoir and the radar antenna was approximately $25 \mathrm{~m}$ above the water surface. Grazing angles were between $3^{\circ}$ and $4.5^{\circ}$ to the centers of the respective range gates. The reservoir depth in the area of the measurements was approximately $3.3 \mathrm{~m}$. The fetch was approximately $1.25 \mathrm{~km}$, with no swell for these experiment conditions.

Figure 4 plots the amplitude and phase histograms for the measured polarimetric scattering data under the low wind condition (data file 144). The wind speed was $2.9 \mathrm{~m} / \mathrm{s}$ and the radar observation direction was approximately crosswind. The amplitude and phase distributions are compared with the theoretical pdf models of (10) and (11). As can be seen from this figure, VV shows a wider amplitude distribution than $\mathrm{HH}$, and the phases appear to be non-uniformly distributed for both 


\begin{tabular}{|c||c|c||c|c|}
\hline File & $144 \mathrm{HH}$ & $144 \mathrm{VV}$ & $160 \mathrm{HH}$ & $160 \mathrm{VV}$ \\
\hline \hline$\langle\rho\rangle$ & $2.18 \times 10^{-4}$ & $4.03 \times 10^{-3}$ & $6.91 \times 10^{-4}$ & $8.65 \times 10^{-3}$ \\
\hline$\sigma_{\rho}$ & $9.59 \times 10^{-5}$ & $2.16 \times 10^{-3}$ & $4.85 \times 10^{-4}$ & $4.63 \times 10^{-3}$ \\
\hline$\frac{\langle\rho\rangle}{\sigma_{\rho}}$ & 2.269 & 1.871 & 1.425 & 1.866 \\
\hline
\end{tabular}

TABLE II

MEAN, STANDARD DEVIATION, AND MEAN-TO-STANDARD DEVIATION RATIO FOR HH AND VV WAVES.
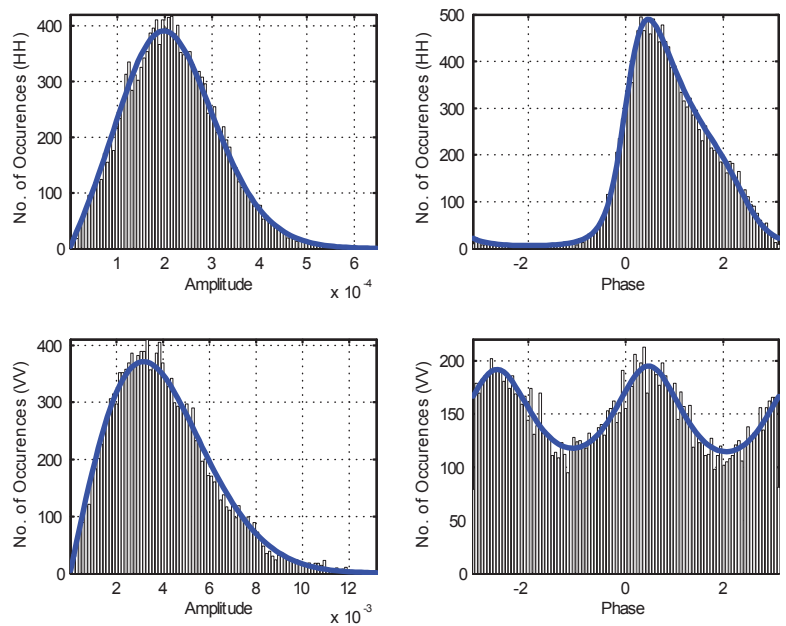

Fig. 4. Amplitude and phase distributions for data file 144 .

HH and VV. The data and model show a fairly good agreement. Figure 5 is for the reservoir data under the moderate wind condition (data file 160). The wind speed was $7.1 \mathrm{~m} / \mathrm{s}$ and the radar observation direction was obliquely upwind. As shown in Figure 5, when the wind speed increases the surface becomes rougher and the phase distribution becomes more uniform. For the amplitude pdf, the measured data also show that VV has a wider distribution than HH. In Table II, we summarize the mean, standard deviation, and mean-to-standard deviation ratio for the amplitude distribution evaluated from the measured reservoir data. Note that the ratio of $\frac{\langle\rho\rangle}{\sigma_{\rho}}$ for the HH polarization of data file 144 is 2.269 , which is larger than 1.913 , while others are smaller.

\section{CONCLUSION}

In this paper we have studied the statistical characteristics of scattering from rough surfaces. Based on the assumption of normal-distributed and correlated real and imaginary scattering components, we derive analytical probability density functions for scattering amplitude and phase. The amplitude pdf is in the form of an infinite sum of modified Bessel functions and the phase pdf is expressed in terms of an error function. The analytic pdf models are functions of the first and second moments of scattered fields: mean, variance, and correlation coefficient, which can be easily evaluated from scattered signals. The analytical amplitude and phase distributions are verified by comparing with both the empirical distributions of simulated data and experimental measurements. Simulated
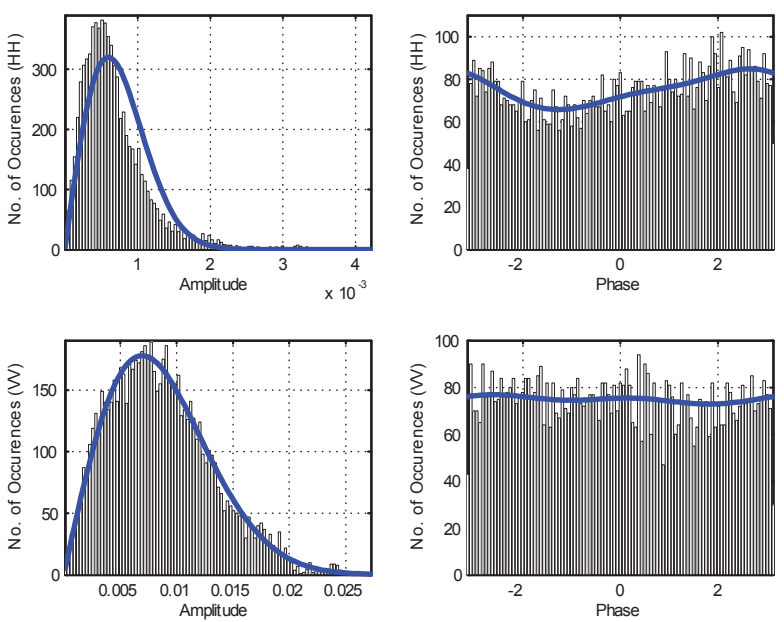

Fig. 5. Amplitude and phase distributions for data file 160 .

data sets were derived from Monte Carlo simulations of bistatic scattering from one-dimensional Pierson-Moskowitz ocean-like rough surfaces. The BMIA technique is applied in solving the Maxwell's equations numerically to compute bistatic scattering signals from rough surfaces. Experimental data were collected from experiments conducted at a windroughened reservoir with an $S$-band polarimetric radar. These simulated and experimental results show that the amplitude and phase pdfs vary with wind speed, observation angle, and polarization. The TM (VV) wave is observed to have a wider amplitude distribution than a TE (HH) wave. The phase of the scattered field is not uniformly distributed, especially under the low wind condition. The analytical amplitude and phase distributions are in good agreement with the empirical distributions of both simulated and experimental data sets.

\section{REFERENCES}

[1] L. Tsang, J.A. Kong, and R.T. Shin, Theory of Microwave Remote Sensing, New York: Wiley, 1985.

[2] P. Beckmann and A. Spizzichino, The Scattering of Electromagnetic Waves from Rough Surfaces, Artech House, 1987.

[3] J.A. Ogilvy, Theory of Wave Scattering from Random Rough Surfaces, Bristol: Adam Hilger, 1991.

[4] L. Tsang, J.A. Kong, K.H. Ding, and C.O. Ao, Scattering of Electromagnetic Waves: Vol.2: Numerical Simulations, New York: Wiley Interscience, 2001.

[5] K.W. Lam, Q. Li, L. Tsang, L. Lai, and C.H. Chan, "On the Analysis of Statistical Distributions of UWB Signal Scattering by Random Rough Surfaces Based on Monte Carlo Simulations of Maxwell Equations," IEEE Trans. Antennas Propagat., Vol. AP-52, pp. 3200-3206, 2004.

[6] K.D. Ward, R.J.A. Tough, and S. Watts, Sea Clutter: Scattering, the K Distribution and Radar Performance, IET, London, 2006.

[7] M. Rangaswamy, "Impact of Diverse Polarisations on Clutter Statistics," IEE Proc.-Radar Sonur Navig., Vol. 153, pp. 30-34, 2006.

[8] A. Farina, F. Gini, M.V. Greco, and L. Verrazzani, "High Resolution Sea Clutter Data: Statistical Analysis of Recorded Live Data," IEE Proc. Radar, Sonar, Navig., Vol. 144, pp. 121-130, 1997.

[9] M.V. Berry, "The Statistical Properties of Echoes Diffracted from Rough Surfaces," Phil. Trans. Roy. Soc. A, Vol. 273, pp. 611-658, 1973.

[10] A. Papoulis, Probability, Random Variables and Stochastic Processes, McGraw-Hill, New York, 1965. 